\section{Scanning memory for multidimensional stimuli with extended practice*}

\author{
JOSEPH DUMAS† \\ State University of New York at Buffalo, Buffalo, New York 14214
}

Memory load and the similarity between positive and negative sets were varied orthogonally in an information-reduction task which required $\mathbf{S}$ to respond either "yes" or "no" to the question of whether or not a visually displayed two-dimensional pattern had been contained in a previously memorized set of patterns. Six Ss were given 8 days of practice. The results showed that throughout practice, there was significant increase in reaction time with increases in the similarity of positive and negative sets. The memory load differences simificantly decreased to zero with practice, and this drop was independent of the similarity effects. It was concluded that extended practice influences memory load differences and effects that reflect the complexity of the memory comparison process differentially.

In 1963, Neiseer, Novick, and Lazar reported finding evidence for parallel procesaing of memorial information in a visual search task. Ss scanned a vertical list of 50 lines, each line containing six alphanumeric characters, for a single target letter at an unpredictable position. As soon as the list appeared, $\mathbf{S}$ began scanning down from the top. When he found a target, S turned a switch which stopped a clock, and the total search time was recorded. Neisser et al found that after about 12 days of practice, the size of the set of items to be searched for $(1,5$, or 10 items $)$ had no effect on the search rate. The model they proposed to account for these results assumes that a categorizing response is a decision which is, in turn, based on the outcome of other lower level tests concerning elementary features of the stimulus. Even for the condition where there was only a single target, a multiplicity of tests is postulated. As stimuli are added to the target set, the number of elementary features which must be tested increases. Thus any increase in search rate associated with increases in memory set size is assumed to be due to these additional feature tests. However, with practice, these feature tests can be performed in parallel, e.g., all the features are examined at the same time.

* Brsed on a disertintion submitted to the araduate school of the State Univerity of New Yotk at Buffalo in partial huliminent of the requiremente for the $\mathrm{PhD}$ dexee. The author wishes to thank Ivine Biedermnn. chrirman of hl discortation committee, Stephen Checkody, and Erwin Segal for their help. The research was undertaken while the author held a traineerhip supported by the U.S. Public Health Service. Acoount No, 60-789A.

tRequest for reprints should be sent to Joseph Dumas, Parchology Department, Oelving Univerdity, Rochester, Michigan 48063.
This finding is in contrast to the results of several studies by Sternberg $(1966,1967)$. In a typical experiment, Ss are shown a small set of items and are asked to memorize them. They are then shown a single item and asked to pull one response lever if the stimulus item was in the list of items (positive response) and another level if it was not (negative response). Sternberg has found that reaction time (RT) is an increasing linear function of set size (M) for both positive and negative responses. This linear function suggests that the addition of an item to the target set has the same effect regardless of the size of the set. The slope of the function relating RT to M thus becomes a measure of the average duration of the time it takes to operate on each item in the set. According to Sternberg, each operation is assumed to consist of a comparison of a representation of the stimulus item with a member of the target set. The slope, therefore, is the average comparison rate. The intercept of the function reflects the combined times of all the other processes involved. These would include stimulus encoding, response selection, and response execution. Sternberg also found that the RT functions for positive and negative responses were parallel. This is consistent with a model in which serial comparison operations are performed exhaustively on each trial.

Recently there have been several studies examining memory search proceses with extended practice in an attempt to resolve the differences between the parallel and serial models (Burrows \& Murdock, 1969; Briggs \& Blaha, 1969). However, one of the problems with testing the implications of the models is that, for the most part, alphanumeric stimuli have been used. Consequently there is little control over the number of features present in the memory sets. This problem can be overcome by the use of multidimensional stimuli that vary in such specifiable characteristics as shape, color, size, etc. Some recent studies using these stimuli have generated results which shed light on the processes involved in a memory search task.

In an experiment by Nickerson (1967), the memory set was defined as a pattern containing three values of three dimensions. Thus, for example, a $S$ might be instructed to respond YES if the stimulus that appeared was large and red and a circle; otherwise he might be instructed to respond NO. Thus the memory set consisted of one stimulus item, and all the other stimuli required a NO response; however, they differed in terms of how many attributes they shared with the memory set stimulus. The number of shared attributes was 0,1 , or 2 . Nickerson found RT to be a direct function of the number of dimensions that needed to be categorized. Thus RTs for NO responses sharing two dimensions with the positive stimulus were slower than RTs for NO responses sharing one dimension, etc. This result rules out any model that depends on the template matching of items. It is still possible, however, to construct a serial exhaustive model in which values within a dimension are searched exhaustively but in which comparisons are self-terminating between dimensions. However, such a model would be inconsistent with the results of Checkosky (1971). He varied both $M$ and the number of dimensions necessary to make a response. Each $S$ was presented, at different times, with two positive sets. One set contained two patterns (e.g., a red circle and a blue square), while the other had three patterns. As in Nickerson's study, the negative set contained patterns with zero (a purple " $x "$ ), one (a blue cross), or two (a red square) values in common with the positive set. Consistent with the results found by Nickerson, the RTs to the negative set were a direct function of the number of relevant values. However, the effects of $M$ were independent of the effects of both dimensionality and response. Such a result suggests that the slope of the function relating $R T$ to $M$ is not reflecting comparison time. However, there is one comparison model which the Checkosky (1971) study does not rule out. Consider the possibility that all items in the memory set are examined exhaustively on each trial but that the time to examine an item is a direct function of its similarity to the stimulus item. Thus the comparison process is self-terminating within items but 
Table 1

Mean Correct RTs and Relative Frequency of Emors (RFE) for the Condensation (C) and Redundant (R) Tasks for $Y$ es and No Responses

\begin{tabular}{|c|c|c|c|c|c|c|c|c|c|c|}
\hline \multirow{2}{*}{$\begin{array}{c}\text { Mernory } \\
\text { Load }\end{array}$} & \multirow{2}{*}{ Task } & \multirow[b]{2}{*}{ Response } & \multicolumn{2}{|c|}{ Block 1} & \multicolumn{2}{|c|}{ Block 2} & \multicolumn{2}{|c|}{ Block 3} & \multicolumn{2}{|c|}{ Block 4} \\
\hline & & & $\mathbf{R T}$ & RFE & RT & RFE & $\mathbf{R T}$ & RFE & RT & RFE \\
\hline $\begin{array}{l}2 \\
I \\
T \\
\mathbf{E} \\
\mathbf{M} \\
\mathrm{S}\end{array}$ & $\begin{array}{l}\mathbf{R} \\
\mathbf{C} \\
\mathbf{R} \\
\mathbf{C} \\
\mathbf{C} \\
\mathbf{C}\end{array}$ & $\begin{array}{l}\text { Yes } \\
\text { Yes } \\
\text { No } \\
\text { No-2 } \\
\text { No-1 } \\
\text { No-0 }\end{array}$ & $\begin{array}{l}.493 \\
.560 \\
.487 \\
.638 \\
.560 \\
.496\end{array}$ & $\begin{array}{l}.064 \\
.046 \\
.045 \\
.042 \\
.000 \\
.000\end{array}$ & $\begin{array}{l}.456 \\
.481 \\
.439 \\
.551 \\
.494 \\
.464\end{array}$ & $\begin{array}{l}.032 \\
.027 \\
.001 \\
.036 \\
.005 \\
.000\end{array}$ & $\begin{array}{r}.438 \\
.483 \\
.436 \\
.526 \\
.485 \\
.445\end{array}$ & $\begin{array}{l}.023 \\
.033 \\
.000 \\
.024 \\
.000 \\
.000\end{array}$ & $\begin{array}{l}.420 \\
.454 \\
.414 \\
.502 \\
.459 \\
.422\end{array}$ & $\begin{array}{l}.031 \\
.064 \\
.000 \\
.046 \\
.005 \\
.000\end{array}$ \\
\hline $\begin{array}{l}3 \\
\mathbf{I} \\
\mathbf{T} \\
\mathbf{E} \\
\mathbf{M} \\
\mathbf{S}\end{array}$ & $\begin{array}{l}\mathbf{R} \\
\mathbf{C} \\
\mathbf{R} \\
\mathbf{C} \\
\mathbf{C} \\
\mathbf{C}\end{array}$ & $\begin{array}{l}\text { Yes } \\
\text { Yes } \\
\text { No } \\
\text { No-2 } \\
\text { No-1 } \\
\text { No-0 }\end{array}$ & $\begin{array}{l}.533 \\
.610 \\
.518 \\
.675 \\
.580 \\
.561\end{array}$ & $\begin{array}{l}.055 \\
.046 \\
.000 \\
.052 \\
.000 \\
.000\end{array}$ & $\begin{array}{l}.478 \\
.512 \\
.463 \\
.578 \\
.494 \\
.495\end{array}$ & $\begin{array}{l}.040 \\
.013 \\
.001 \\
.046 \\
.000 \\
.000\end{array}$ & $\begin{array}{r}.455 \\
.484 \\
.446 \\
.532 \\
.487 \\
.458\end{array}$ & $\begin{array}{l}.018 \\
.045 \\
.002 \\
.031 \\
.000 \\
.000\end{array}$ & $\begin{array}{l}.418 \\
.450 \\
.422 \\
.507 \\
.457 \\
.431\end{array}$ & $\begin{array}{l}.018 \\
.046 \\
.002 \\
.046 \\
.005 \\
.000\end{array}$ \\
\hline
\end{tabular}

exhaustive between items. A stimulus item that had one attribute value in common with one of the memory items would be responded to more slowly than a stimulus item that did not share any attribute values with any of the memory items. However, this difference would be independent of $M$. This model postulates a process in which information about whether or not a match has occurred can be used to terminate the further examination of an item but not to terminate the examination of subsequent items. It is, however, consistent with the results of Checkosky (1971).

The present experiment manipulates the same variables as Checkosky but with extended practice. Besides replicating Checkosky for the first 3 days this manipulation allows for a further test of the independence of comparison complexity and $M$. It also provides for a test of the parallel model. Neisser et al (1965) explain the elimination of $\mathbf{M}$ effects by assuming that feature tests are being carried out in parallel. This model predicts that the effects of dimensional matching and the efiects of the size of the memory set vary together. If the effects of $M$ disappear with practice, the effects due to the number of feature tests must disappear at the same time. Similarly serial exhaustive comparison models explain $M$ effects as being due to the number of serial comparisons which must be carried out. Thus if the effects of the number of comparisons remain with extended practice, the effects of $M$ must also remain, since the more patterns there are, the more comparisons there will be. The one result which none of these models can handle is one in which the effects of the number of dimensions which need to be matched remain while the effects of $M$ disappear.

\section{METHOD Design}

Two factors, (1) the size of the positive set (M) and (2) the similarity of the negative to the positive set, were varied factorially over 8 days in a memory search task. $M$ was varied by having positive sets of either two two-dimensional patterns (e.g., a blue square and a red circle) or three two-dimensional patterns. Similarity was manipulated independently of $\boldsymbol{M}$ by having $S$ perform two different experimental tasks. The tasks differed in how many attributes the positive and negative sets shared. In the redundant task, none of the attributes of the positive set patterns were contained in the negative set patterns. For example, if the positive set was a red circle and a blue square, the patterns requiring a NO response would be neither red nor blue nor circles nor squares (e.g., a yellow triangle and a green "X"). In the condensation task, the patterns requiring a NO response could vary in the number of attributes they shared with positive set patterns. There were three levels of similarity. Each negative set contained patterns sharing two, one, or no attributes with positive set patterns. For example, if the positive set consisted of a red circle and a blue square, the negative set would contain patterns which shared two attributes with the positive set (e.g., red square, blue circle), patterns which shared one attribute with the positive set (e.g., yellow square, blue " $X$ "), and patterns which shared no attributes with the positive set (e.g., yellow triangle, green "X"). These three types of negative-set patterns will be referred to as NO.2, NO-1, and NO.0 patterns, respectively.

The combination of two conditions and two values of $M$ resulted in four experimental conditions. Ss participated in all conditions on each of 8 days, separated by at least 1 day but never more than by 2 . Three Ss pressed the positive response key with their preferred hand and three with their nonpreferred hand.

\section{Subjects}

The Ss were six female State
University of New York at Buffalo undergraduates who were paid for their participation.

\section{Apparatus}

Stimuli were generated by an in-line display (Industrial Electronic Engineers, Inc., Model 10. $0 \mathrm{k} 21-1820-1$ ) set on a $3 \times 3 \mathrm{ft}$ gray wooden frame. The experimental cubical was dimly lit. Stimulus selection was performed manually by the $E$ through a panel of switches. The pre- and postexposure fields as well as the background field were uniformly black. 'Ss' index fingers rested on plastic keys which, when pressed, activated a microswitch. RTs were measured by a Lafayette Standard Electric timer accurate to 1/100th sec. $A$ set of lights on the panel in front of $E$ indicated S's response. The sequence of ready-signal presentation and reset was controlled by an electronic timer (Lafayette Model 14314a). RT and response (YES vs NO) were recorded manually by $E$.

\section{Stimuli}

The stimuli consisted of white outlines on colored backgrounds. The colors were background circles, 1 in. in diam, with hues of red, green, yellow, purple, blue, and gray. The six shapes were outlines of a circle, square, triangle, dash (a horizontal line segment), " $x$," and "**." These shapes were clear and were centered inside the colored circles.

\section{Procedure}

On each day, four Ss were assigned randomly to four sequences of a Latin square that balanced both order and sequence effects. Because there were six $\mathrm{Ss}$ and four conditions, it was not possible to balance practice completely. The remaining two Ss were assigned randomly on odd-numbered days to two of the four sequences. On even-numbered days, the two remaining sequences were assigned to the remaining Ss. All patterns used occurred in all conditions an equal number of times.

Before every condition on every day, each $\mathbf{S}$ was shown the positive and negative sets. On each day, instructions stressing accuracy relative to speed were read. The sequence on each trial consisted of a $0.5-\mathrm{sec}$ warning buzzer, followed 1 sec later by a 1-sec stimulus presentation. Ss were given feedback about accuracy and speed for each response. The interstimulus interval was $8 \mathrm{sec}$ on the first day, $7 \mathrm{sec}$ on the second day, and $6 \mathrm{sec}$ on the remaining days. Each session was made up of four conditions (Condensation Memory Load 2, Condensation Memory Load 3, Redundant Memory Load 2, 


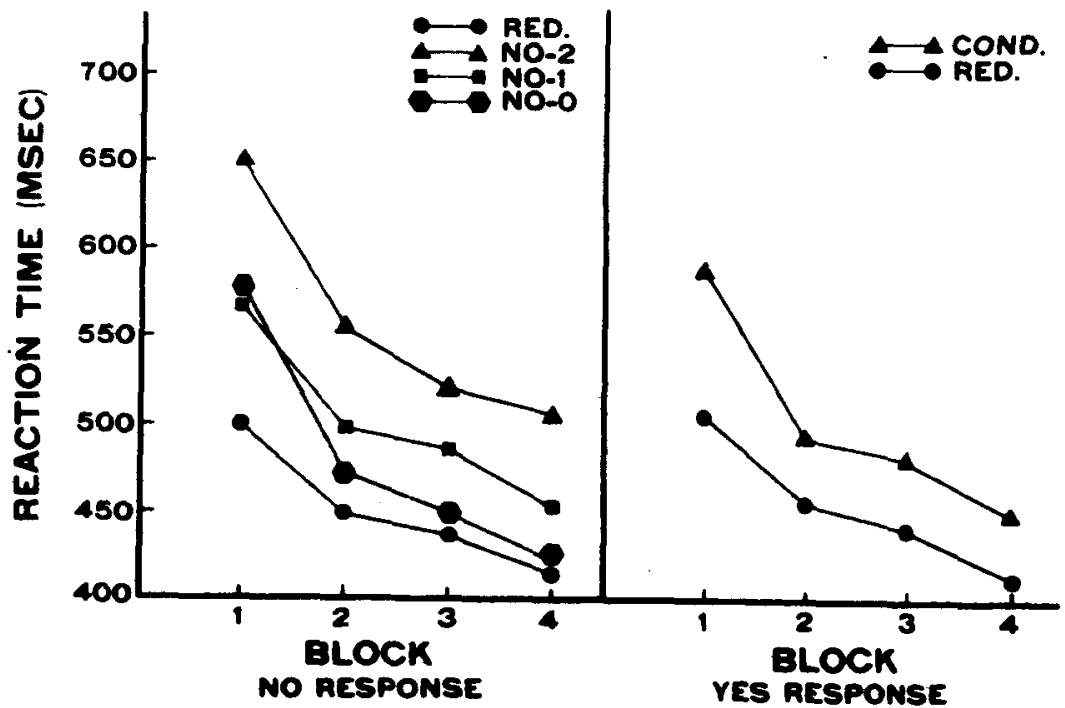

Fis. 1. Menn correct RTs as a function of blocks for redundant (RED.) and condenation (COND.) YBS reponses and for redundant and condencation NO-2, NO-1, and NO-O NO responses.

and Redundant Memory Load 3) with four blocks each. Each block within conditions consisted of the presentation of 18 stimuli, 6 of which were positive-set patterns. For the redundant conditions, the 12 remaining stimuli had no values in common with the positive set. For the condensation condition, the 12 remaining stimuli were made up of four NO-2s, four NO-1s, and four NO-0s. Thus each $S$ responded to 72 stimuli under each condition per day. For the data analyses, the RTs to the first 18 stimuli for each condition were excluded and the correct RTs for each successive block of 2 days were combined. A count of the total response errors was tabulated separately for positive and negative responses for each condition to provide a relative frequency of error (RFE).

\section{RESULTS}

The mean RTs for each response and memory-load condition for both the redundant and condensation conditions for each block of days, along with the RFE, appear in Table 1. It is clear that the accuracy instructions were successful in generating high response accuracy.

Figure 1 shows the correct RTs plotted for each condition collapsed over memory load for each block. First looking at the YES responses, the condensation RTs were significantly slower $[F(1,5)=110.86, p<.01]$ than the redundant $R T s$. Secondly, the condensation NOs differed significantly from each other. The NO-2s were slower than the NO-1s $[F(1,5)=7.38, p<.05]$, and the NO-1s were slower than the NO-Os
$[F(1,5)=10.09, p<.05]$. In addition, the difference between the NO-1s and NO-Os interacted with blocks $[F(1,5)=6.98, p<.05]$. Thus the RT differences assumed to be due to matching complexity were significant and remained so throughout the four blocks of practice. However, none of these differences significantly interacted with memory load.

Figure 2 presents the differences between the two- and three-item memory sets over blocks for the conditions of Fig. 1. As can be seen, all of the memory-load effects drop with practice. Statistically each of the conditions, except for the condensation NO-18, interacts significantly with blocks. The $F(3,15)$ values for the condensation and redundant YES responses were 4.39 $(p<.05)$ and $4.54 \quad(p<.05)$ respectively. For the redundant NO responses, the value was 5.44 $(p<.01)$. For the condensation $\mathrm{NO}-2 \mathrm{~s}, \mathrm{NO}-1 \mathrm{~s}$, and $\mathrm{NO}-0 \mathrm{~s}$, the values were $4.38(p<.05), 0.04$, and 6.25 $(p<.01)$, respectively. In addition, a eparate analysis was calculated for each condition on Block 4 to teat whether the memory load effects differed from zero. None of the F values were simificant at the .05 level.

\section{DISCUS8ION}

The results on Blocks 1 and 2 of the present experiment replicate the findings of Checkosky (1971) in all but one point. The condensation YESs were slower than the redundant YESs, and the NO-2s were slower than the NO-1s, which were slower than the NO-0s. Furthermore, these effects were independent of $M$. The difference between the condensation NO-Os and the redundant NOs on Block 1 was not expected. However, it is probably due to a peculiarity in the stimuli. For two of the S6, one of the condensation NO-0 patterns happened also to be a redundant YES pattern. For those Ss, RTs to this pattern, when it appeared as a NO-0, were slower than to the

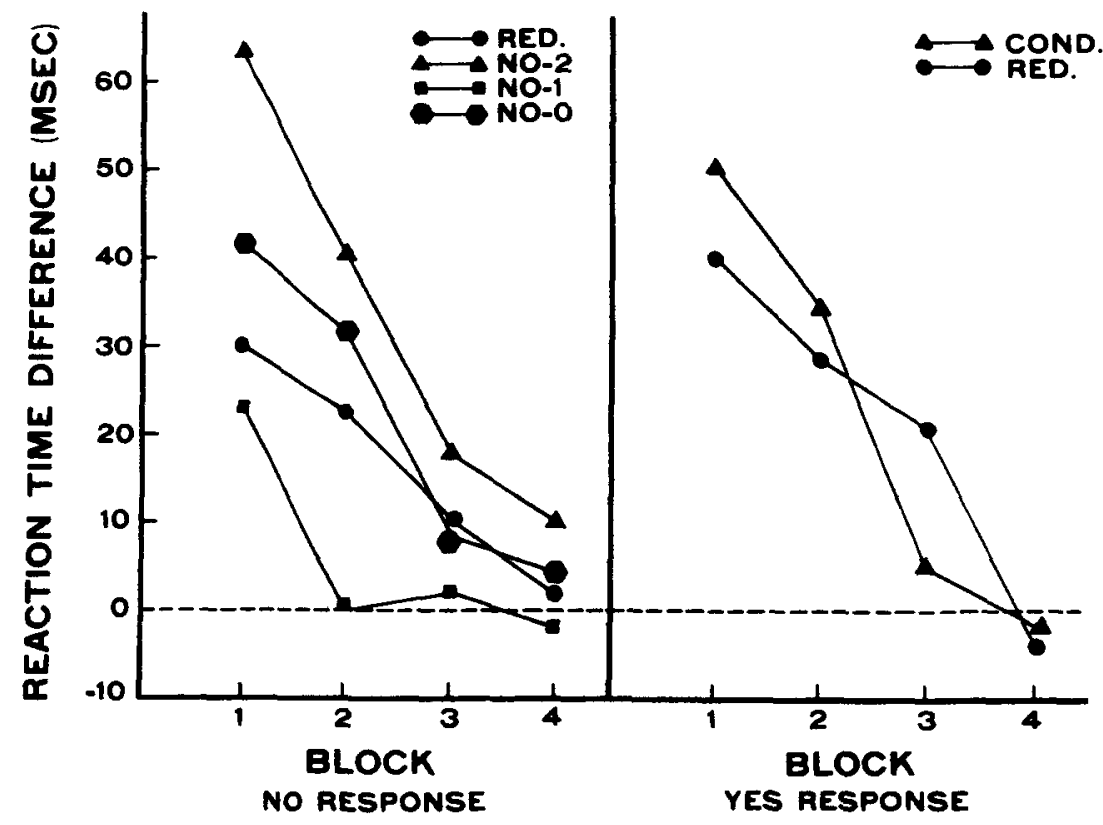

Fig. 2. Mean difference between memory-load conditions as a function of blocks for redundant (RED.) and condensation (COND.) YES responses and for redundant and condensation $\mathrm{NO}-2, \mathrm{NO}-1$, and $\mathrm{NO}-0 \mathrm{NO}$ responses. 
other NO-0 pattern on Block 1.

The pattern of results of the present study suggests that processes that are dependent on $M$ and processes that are dependent on the number of attribute matches may be occurring at different stages. Attribute differences do not effect the slope of the functions relating $R T$ to $M$. Since, in the condensation task, $S$ does not know how many dimensions will be needed to determine the correct response, he must encode both dimensions of a stimulus. Thus, though dimensional effects show up in the intercept, they cannot be reflecting differences in encoding time. These findings are inconsistent with the memory-scanning model proposed by Sternberg (1967). While that model has not been specifically applied to multidimensional stimuli, it has been tested with letters, numbers, nonsense forms, and photographs of faces (Sternberg, 1969).

Of the models considered above, the only one which is consistent with the present results is the model which postulated self-terminating comparisons within items but exhaustive comparisons between items. This model could account for the present results, assuming that, with practice, all of the items in the memory set are examined at the same time but that the comparisons are still affected by the similarity of the stimulus attributes to the memory-set attributes. The alternative that Checkosky (1971) proposed, namely, that $M$ efferts reflect the time for the retrieval or generation of the memory set, also remains tenable. However, it must assume that, with practice, the memory set need not be retrieved on each trial but can remain generated throughout a block of trials.

The finding that the dimensional effects remain after 8 days of practice while the memory-load difference is reduced to zero is also inconsistent with the parallel feature-testing model. It attributes the early $M$ effects to differences in the number of feature tests and their gradual reduction to the growing ability to perform feature tests in parallel. In the present study, RT differences reflecting the complexity of feature tests were independent of $M$ effects. While the curves in Fig. 1 do not appear to have reached asymptote, the Neisser et al (1963) model predicts that the $M$ effects and the dimensional differences should vary together. Since the $M$ effects are no longer present after 8 days, the dimensional differences should also have disappeared. The fact that they did not is inconsistent with the model.

\section{REFERENCES}

BRIGGS, G.. \& BLAHA, J. Memory retrieval and central comparison times in information processing. Journal of Experimental Psychology, 1969, 79, Experime

BURROWS, D., \& MURDOCK, B. B. Effects of extended practice on high-speed scanning. Journal of Experimental Psychology, 1969, 82. Experiment

CHECKOSKY, S. Speeded classification of multidimensional stimuli. Journal of Experimental Psychology, 1971, 87, 383-388.

NEISSER, U., NOVICK, $R$.. \& LAZAR, R. Searching for ten targets simultaneously. Perceptual \& Motor Skills, 1963, 17. 427-432.

NICKERSON, R. S. Categorization time with categories defined by disjunctions and conjunctions of stimulus attributes. Journal of Experimental Psychology. 1967, 73, 211-219.

STERNBERG, S. High-speed scanning in human memory. Science, 1966, 153 , 652-654.

STERNBERG, S. Two operations in character-recognition: Some evidence from reaction-time measurements. Perception \& Psychophysics, 1967, 2, 45-53.

STERNBERG, S. Memory-scanning: Mental processes revealed by reaction-time experiments. American Scientist, 1969, 57, 421-457.

(Accepted for publication September 23, 1971.) 